\title{
Composite electrode of carbon nanotubes and vitreous carbon for electron field emission
}

\author{
E. Y. Matsubara, ${ }^{1}$ J. M. Rosolen, ${ }^{1, a)}$ and S. Ravi P. Silva ${ }^{2}$ \\ ${ }^{1}$ Departamento de Química-FFCLRP, Universidade de São Paulo, Ribeirão Preto, São Paulo \\ SP14040-901, Brazil \\ ${ }^{2}$ Nano-Electronics Centre, Advanced Technology Institute, University of Surrey, Guildford GU2 7XH, \\ United Kingdom
}

(Received 13 September 2007; accepted 27 June 2008; published online 4 September 2008)

In this work, the electron field emission behaviour of electrodes formed by carbon nanotubes (CNTs) grown onto monolithic vitreous carbon (VCarbon) substrates with microcavities is presented. Scanning electron microscopy was used to characterize the microstructure of the films. Tungsten probes, stainless steel sphere, and phosphor electrodes were employed in the electron field emission study. The CNT/VCarbon composite represents a route to inexpensive excellent large area electron emission cathodes with fields as low as $2.1 \mathrm{~V} \mu \mathrm{m}^{-1}$. In preliminary lifetime tests for a period of about $24 \mathrm{~h}$ at an emission current of about $4 \mathrm{~mA} \mathrm{~cm}^{-2}$, there is an onset degradation of the emission current of about $28 \%$, which then stabilizes. Electron emission images of the composites show the cavity of the samples act as separate emission sites and predominantly control the emission process. The emission of CNTs/VCarbon was found to be stable for several hours.

(C) 2008 American Institute of Physics. [DOI: 10.1063/1.2974090]

\section{INTRODUCTION}

Carbon nanotubes (CNTs) consisting of $s p^{2}$-hybridized carbon atoms are cylindrical structures with a diameter ranging from $1 \mathrm{~nm}$ to several tens of nanometers and length scales from tens to hundreds of micrometers. High electronic conductivity and chemical stability, together with good mechanical strength, are some of the excellent features of CNTs. Because of these unique properties, there is a belief that the field emission display (FED) technology making use of CNTs as electron emitter could be a threat to the dominance of liquid crystal displays in the flat-panel display arena, provided suitable cathode materials can be produced. ${ }^{1}$ The FED is the technology of choice for ultrahigh definition, wide-screen displays and televisions. ${ }^{2-5}$ However, the effective use of CNTs is yet to be established and is currently a significant challenge. FED manufacturing requires CNT to be grown in precise positions to predetermined sizes and densities; height, diameter, and tip sharpness affect emission voltage, while density affects current. ${ }^{6}$ One drawback is the degradation during emission associated with the electronic resistance at the interface between the carbon tubes and the substrate, which restricts their use in commercial emission devices. $^{7,8}$ The presence of heating at the film/substrate interface generates different mechanical stresses on the film, which leads to an increase in the resistance and/or the voltage barrier damage during the electron emission process over extended periods.

Recently, an electrode structure composed of composites of CNTs based on carbon substrates with a three-dimensional (3D) structure ${ }^{9-11}$ has been proposed as a route to avoiding the above-mentioned drawbacks. In this paper we present yet

\footnotetext{
${ }^{a)}$ Author to whom correspondence should be addressed. Electronic mail: rosolen@ffclrp.usp.br. FAX: + 55166338151.
}

another 3D textured cathode combining CNTs and vitreous carbon (VCarbon) having microcavities to obtain a reproducible electron emission current at low fields. The carbonbased composites were developed by growing the CNTs onto VCarbon substrates having microcavities.

\section{EXPERIMENTAL SECTION}

In this work the CNTs were mounted on the surface of monolithic VCarbon via the decomposition of methanol on metallic particles of cobalt and manganese. The anchoring of the catalysts on the VCarbon was carried out by dipping the felt into an equimolar $\mathrm{Co} / \mathrm{Mn}$ acetate solution, followed by drying at $80{ }^{\circ} \mathrm{C}$ under ambient conditions. Reduction in the acetate salts to the corresponding metals occurred during the heating of the felt under $\mathrm{N}_{2}$ atmosphere, which was the gas used to transport the methanol vapor at $50{ }^{\circ} \mathrm{C}$ to the oven at a flow rate of $0.2 \mathrm{ml} \mathrm{min}^{-1}$. The CNT growth temperature and time were $650{ }^{\circ} \mathrm{C}$ and $20 \mathrm{~min}$, respectively. To remove the catalysts from the tips of the tubes or the catalysts that were not active for methanol decomposition, the electrodes were submitted to concentrated $\mathrm{HCl}$ treatment under reflux and magnetic stirring for $6 \mathrm{~h}$ and then dried under vacuum at $100{ }^{\circ} \mathrm{C}$. The mass of CNTs in the composite was $45.8 \mathrm{mg}$.

The emission characterization was carried out using a FE scanning electron microscope (FE-SEM) having a setup for electrical characterization, that is, a stainless steel sphere anode $(0.5 \mathrm{~cm}$ diameter $)$ with vacuum chamber of about 2 $\times 10^{-6}$ Torr. The distance between the semisphere anode and the CNT/VCarbon cathode was controlled by a stepper motor with a minimum step of $2.5 \mu \mathrm{m}$. The emission of the electrode was also studied using a phosphor electrode under similar vacuum, and two Teflon foils (500 $\mu \mathrm{m}$ thick) were used as separators between CNT/VCarbon and the phosphor electrode. The electrical contact of CNT/VCarbon was 

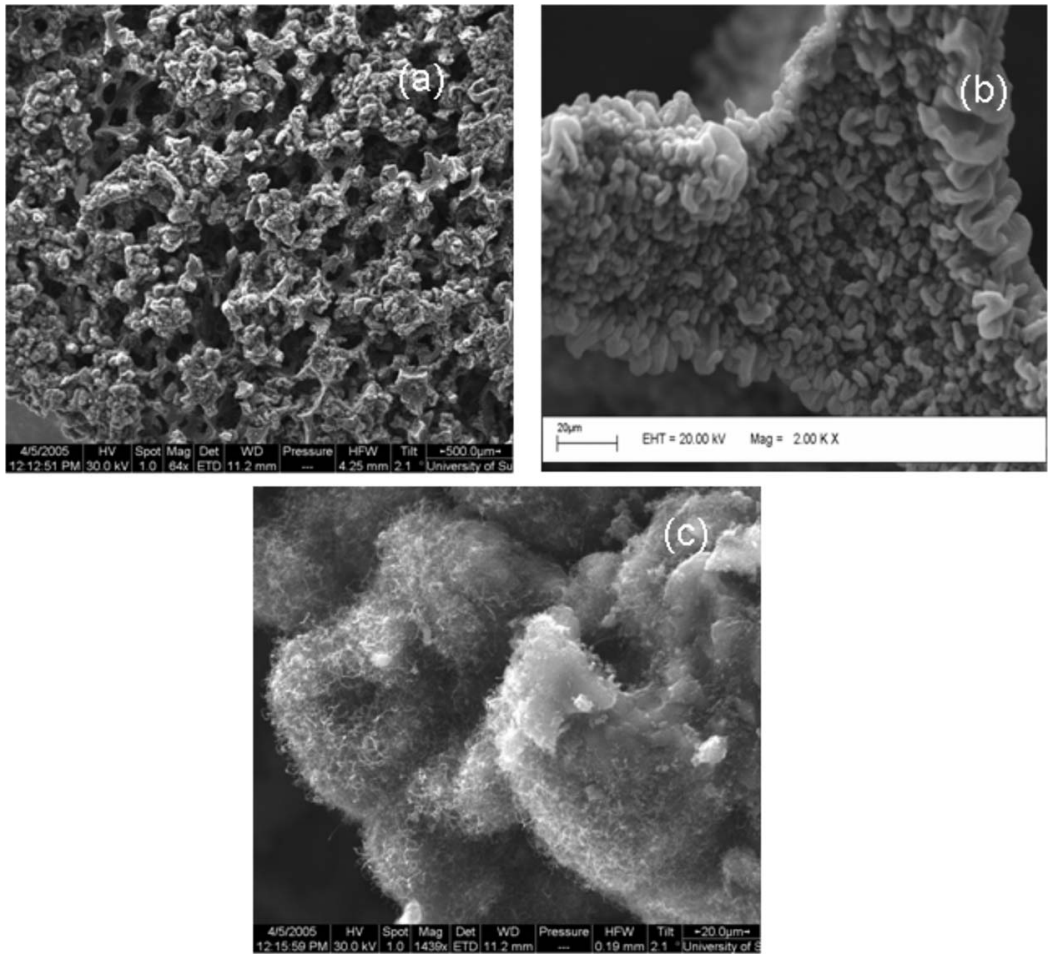

achieved by using a thin layer of silver glue. The composite had a square surface area $\left(0.8 \times 0.8 \mathrm{~cm}^{2}\right)$ and thickness of about $0.5 \mathrm{~cm}$.

\section{RESULTS AND DISCUSSION}

Figure 1 shows microimages for the VCarbon and a typical CNT/VCarbon electrode acquired using the FE-SEM microscope. The SEM pictures show that there are large amounts of CNTs with a cotton-like appearance mainly on the outer surface of the VCarbon substrates [Fig. 1(a)]. It is possible to see that the CNTs abundantly cover the walls of the majority of the cavities [Fig. 1(b)]. In some CNTs it is possible to see a dense agglomeration of tubes, with large deposits of cotton-like CNTs [Fig. 1(c)]. The SEM pictures suggest that catalyst dispersion used for CNT growth was able to concentrate a large number of catalytic particles on the outer surface of VCarbon, thus providing many sites for CNT growth. The cotton-like appearance of these deposits also indicates the presence of a large quantity of amorphous carbon. In fact, it is well known that amorphous carbon in CNT samples are often found as a binder connecting the CNTs, as can be observed in a work devoted to the study of CNT purification. ${ }^{12}$ Another aspect that should be mentioned regards the CNT types that are expected for this sample. For the CNT growth conditions applied in the preparation of the composite studied in this work, the presence of cup-stacked nanostructures has been observed systematically. ${ }^{11}$ A systematic study shows the influence of catalyst dimension as well as reasons as to why the methanol is able to yield this type of nanostructure.

Figure 2 shows the behavior of the FE current for typical cotton-like CNT deposits found in Fig. 1, probed by a tungsten tip assembled inside of the chamber of SEM. In this setup, the composite was grounded using a silver epoxy.
FIG. 1. SEM pictures of a typical CNT/VCarbon electrode and a typical cotton-like CNT deposit on the surface of carbon substrate.
The emission curves of cotton-like CNTs shown in Figs. 3(a) and 3(c) are similar even when the deposit undergoes a small rotation and the gaps are different (Fig. 2). When only the gap distance is increased [Figs. 3(b) and 3(c)], changes in the threshold field $E_{\text {th }}$ (emission current of $1 \mathrm{nA}$ ) are observed, whose values are found at about 25-35 $\mathrm{V} \mathrm{\mu \textrm {m } ^ { - 1 }}$ (Fig. 2). In addition, hysteresis on the $I$ - $V$ curves (Fig. 2), assigning the difference in the applied fields between the upward and downward voltage cycles, disappears when the gap decreases from 8.2 to $6.7 \mu \mathrm{m}$.

Figure 2 shows that in the cotton-like deposits, the emission occurs at very high $E_{\text {th }}$ because of the screening effect from the denser CNTs. Another interesting aspect is that for the gaps 5.4 and $8.2 \mu \mathrm{m}$ [Figs. 3(b) and 3(c), respectively], the emission current versus the applied voltage is similar, suggesting that the enhancement factor in the cotton-like deposit is not constant since at $x=8.2$ and $5.4 \mu \mathrm{m}$ the $E_{\text {th }}$ are identical.

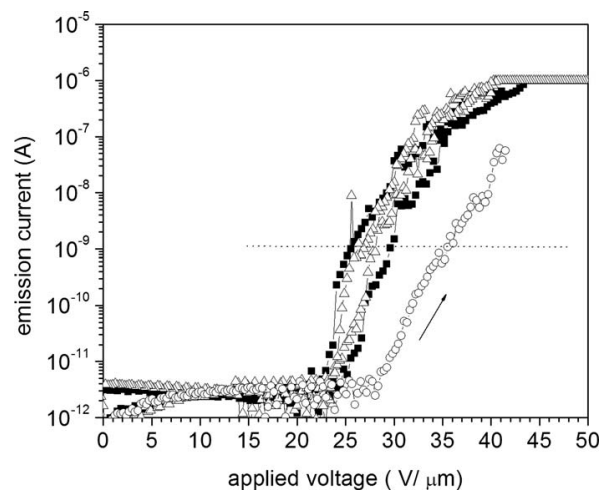

FIG. 2. FE current vs voltage curves of typical cotton-like CNT deposits found on the cavities of the CNT/VCarbon composite collected with W tip inside the SEM at gaps of $5.4(\triangle), 6.7(\bigcirc)$, and $8.2 \mu \mathrm{m}(\mathbf{\square})$. 

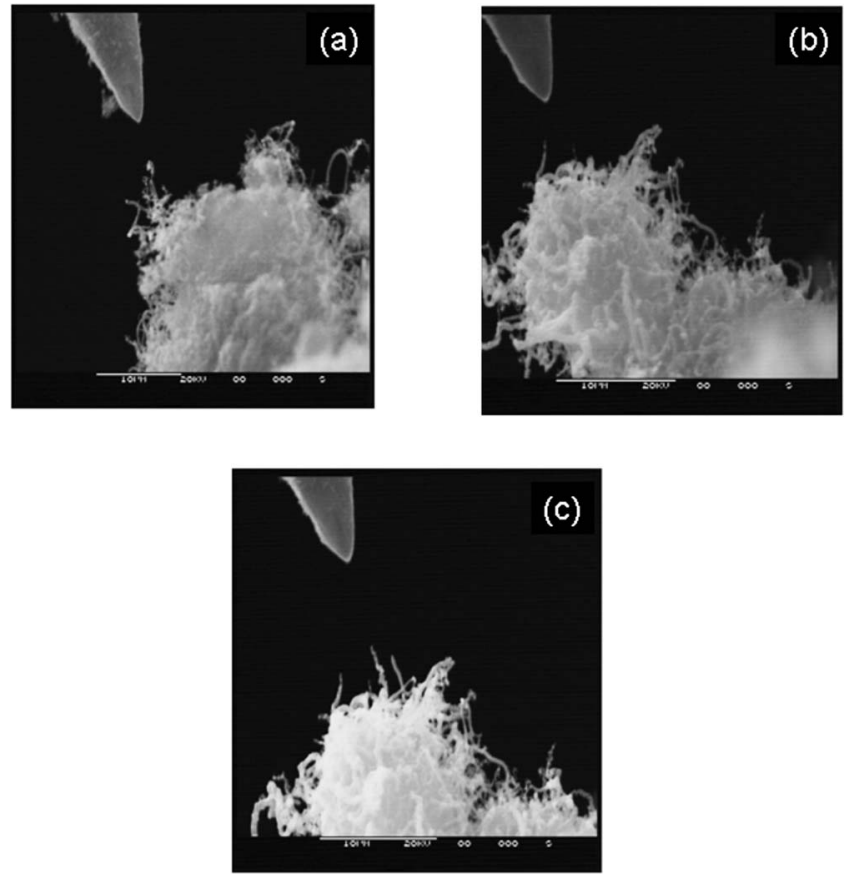

FIG. 3. SEM pictures corresponding to the emission curves shown in Fig. 2.

Typical FE current curves of the CNT/VCarbon composite and VCarbon alone probed by using a semisphere are shown in Figs. 4(a) and 4(b), respectively. The CNT/ VCarbon composite is able to provide a current of $1 \mathrm{nA}$ at a

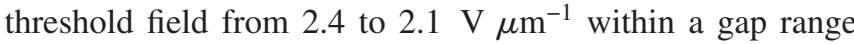
from 200 to $400 \mu \mathrm{m}$. The CNT/VCarbon curves also present

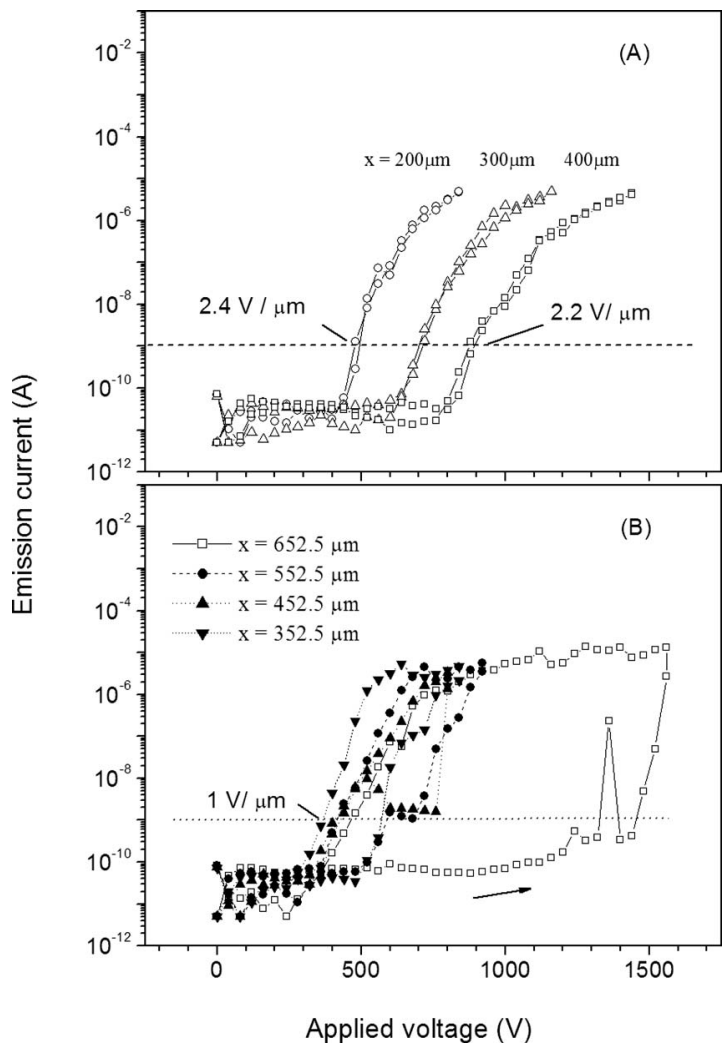

FIG. 4. FE current and voltage cycles of (a) CNT/VCarbon and (b) VCarbon.

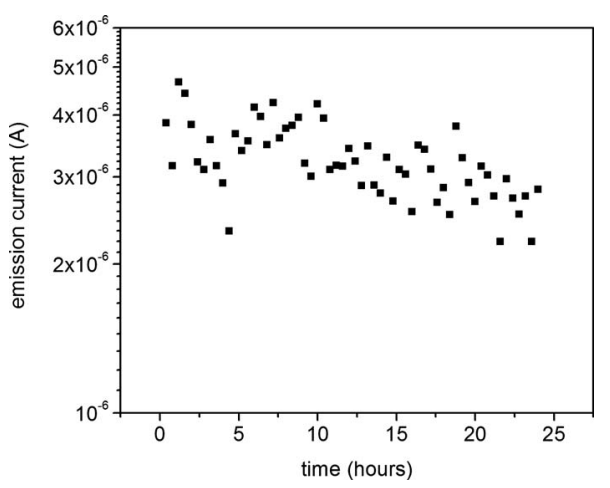

FIG. 5. Stability of the FE current of CNT/VCarbon for a period of $24 \mathrm{~h}$.

a very low hysteresis effect. On the other hand, VCarbon

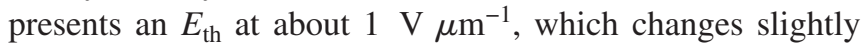
from 352.5 to $552.5 \mu \mathrm{m}$, but has a large hysteresis mainly when the gap distance reaches $652.5 \mu \mathrm{m}$. Therefore, the VCarbon substrate appears to give unstable emission probably due to its geometry, which has many edges and corners that should have a very high enhancement factor. In fact, for smaller gaps than those shown in Fig. 4(b), it was impossible to measure the emission because the samples generated many electrical sparks, clearly showing very high current densities being the sources from the cathodes.

These results show that the $I-V$ curve of CNT/VCarbon is due to the contribution from two emitters: the VCarbon substrate and the CNTs on the surface and cavities of the monolithic VCarbon. However, in the CNT/VCarbon composite the CNT seems to play a significant role since its presence completely changed the emission behavior of the VCarbon substrate. This suggests that the CNTs are probably functioning as emission channels for electrons extracted from the VCarbon.

The stability of the CNT/VCarbon composite studied in Fig. 4 was also probed as a function of time. Figure 5 shows the behavior of the emission current of CNT/VCarbon versus time at $800 \mathrm{~V}$ for a gap distance of $200 \mu \mathrm{m}$ using the sphere-to-plane geometry. During a period of $24 \mathrm{~h}$, it was possible to see that the CNT/VCarbon electrode underwent current variations. When the experiment was interrupted, the current was about 28\% lower than in the first point (Fig. 5). We believe that this variation is mainly associated with the elimination of impurities in the sample (e.g., amorphous carbon, functional groups). The electrical contact between the CNT and the surface of the carbon substrate should have a small electrical resistance since both materials are carbonbased.

Finally, Fig. 6 shows the image of the electron FE of a CNT/VCarbon disk probed by the phosphor counterelectrode at increasing applied voltage (gap of $1 \mathrm{~mm}$ ) after the stability test was carried out (Fig. 5). The phosphor CNT/ VCarbon display has a geometric area of about $0.7 \mathrm{~cm}^{2}$, assembled with a gap of $1 \mathrm{~mm}$. At $800 \mathrm{~V}$ only a small bright point on the phosphor screen can be seen. With increasing field, other points are observed and the previous ones have an increased brightness. It is worth noting that the sites have a similar shape, resembling the cavities of VCarbon depicted in Fig. 1. This means that the emission in the CNT/VCarbon 

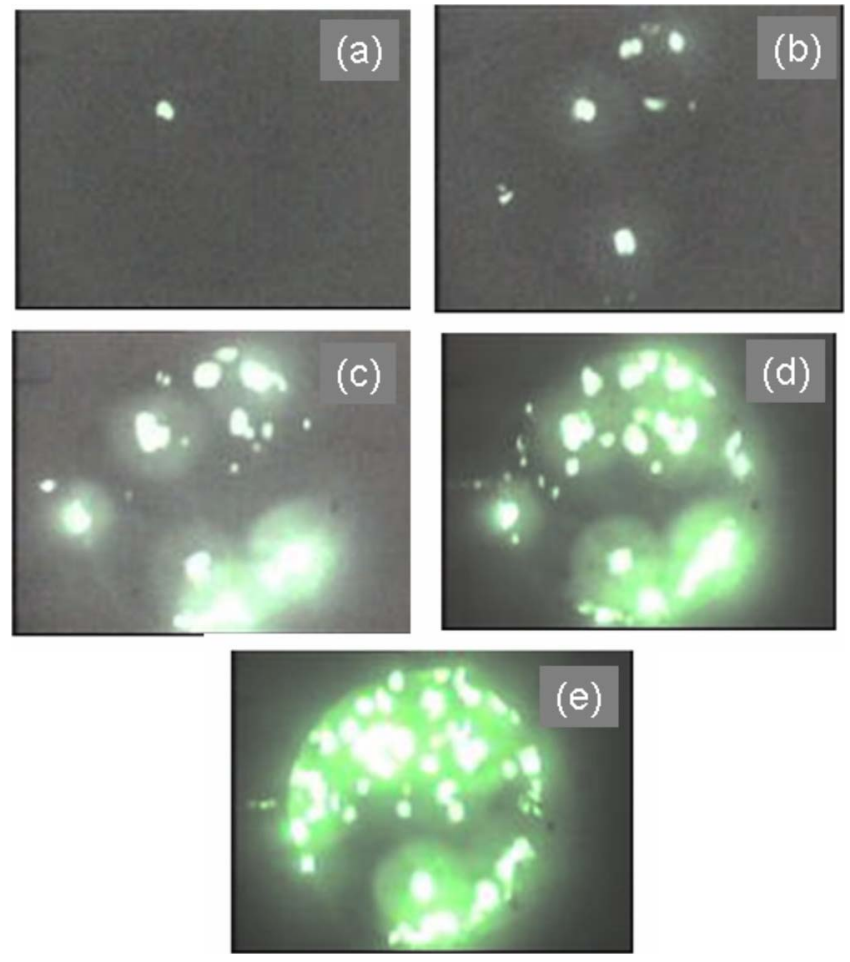

FIG. 6. (Color online) Electron FE pictures of a CNT/VCarbon disk probed by the phosphor counter-electrode at increasing applied voltage (gap of $1 \mathrm{~mm}$ ). The voltages applied on the display are (a) 1000, (b) 1250, (c) 1500 , (d) 1750 , and (e) $2000 \mathrm{~V}$.

composite is determined by several emission sites whose properties depend probably on the amount of CNTs on each cavity. In Fig. 2 it can be seen that the electron emission in the cotton-like CNTs (high concentration of nanotubes) occurs at much higher $E_{\text {th }}$ compared with well-dispersed CNTs or less concentrated nanotubes. The cavities that contain a smaller concentration of CNTs on their surfaces should emit an effective field lower than that of the sites rich in high density, cotton-like CNTs. Thus, we believe that the cavities of CNT/VCarbon are the main emission sites in the sample because of the differences between the $E_{\text {th }}$ values of the cotton-like and composite samples (Figs. 2 and 5). In the cotton-like deposits with high concentration of CNTs and amorphous carbon, the screening effect is expected to be larger than in the cavity sites.

The mechanism of electron emission in the CNT/ VCarbon electrode is very interesting because both carbon of composite are electron emitter. The VCarbon on its own is a very good emitter as seen in the data. However, the data also show the emission is not steady and has fluctuations. Now, in the case of the CNT grown on VCarbon, a larger beta factor due to the CNT growth on VCarbon in places should be expected, and some of the sharper tips of the VCarbon will have also vaporized. So, though a higher effective beta, the very high betas associated with the substrate would be removed. Now, if this is the case as suggested, the gap distance-angular coefficient ratio would expect steadier and more stable current, and maybe some sacrifice to the current density due to the increased steady state emitting surfaces, each screening the other, and therefore also a decrease in the threshold field. This decrease in the threshold field for higher current emitting surfaces was observed. ${ }^{13}$ This could explain the increase in the threshold field and almost steady current density. The higher beta factors for CNT on CNT were described. Secondary nanotube growth on aligned carbon nanofiber arrays for superior FE. ${ }^{14}$ This kind of mechanism seems to be supported by the behavior of slope of $\ln \left(I / V^{2}\right)$ versus $1 / V$ in Fig. 4, where $I$ is emission current and $V$ is the applied voltage (Fowler and Nordheim model). The enhancement factor is proportional to the gap distance-angular coefficient ratio. For CNT/VCarbon this ratio changes a little with an increase in the anode-cathode distance, while for VCarbon the ratio, whose emission current is unstable, it presents a maximum at $452.5 \mu \mathrm{m}$. This ratio is about 0.023 , 0.020 , and $0.021 \mu \mathrm{m}$ at 200,300 , and $400 \mu \mathrm{m}$ to CNT/ VCarbon and 0.029, 0.082, 0.057, and $0.069 \mu \mathrm{m} \mathrm{V}$ at 352.5, $452.5,552.5$, and $652 \mathrm{~mm}$ to VCarbon.

\section{CONCLUSIONS}

In this work CNT/VCarbon composites were prepared and shown to have good cold FE electrode properties. It is not an easy task to determine the emission area for the CNT/ VCarbon composite due to its complex structure. Although there are no conditions to provide an accurate value of the current density of the CNT/VCarbon electrodes, we have used the equation proposed in Refs. 12 and 15, which gave us an effective emission area of about $11 \times 10^{-4} \mathrm{~cm}^{2}$ at $200 \mu \mathrm{m}$. By using this area value, the CNT/VCarbon samples provided an emission of about $4 \mathrm{~mA} \mathrm{~cm}^{-2}$ (Fig. 5). However, we believe that the real emission area of our electrode is smaller than that determined using the equation $A$ $=2 \pi R d\left(2^{1 / n}-1\right)$, where $A$ is the emission area, $n \sim 18, R$ is the radius of the anode $2.5 \mathrm{~mm}$, and $d$ is the anode-cathode separation. The conversion of the sphere area into a planar surface is a good approximation for flat electrodes based on thin films. The electrode studied here presents several cavities filled partially by CNTs, which is, therefore, a very different situation that is found in large area planar measurement configurations, where often it is used as higher threshold fields for probing FE measurements. In fact, when flat electrodes are probed in the same setup, which was used to study the CNT/VCarbon, higher emission current density can be obtained without burning of the phosphor electrode or overloading of current detection, as what occurred in the case of the composite. Thus, the composite prepared in this work could open unconventional routes for the development of electron FE devices. The fact that the composite cavities act predominantly as emitter sites is an interesting result since it could to be used to mount nanotubes with the aim of decreasing the screening effect. In this sense, the CNT loading is a key factor to improving the composite performance for electron FE devices. A better dispersion of CNTs in the cavities should reduce the $E_{\mathrm{th}}$ of the composite, as observed for the CNT/felt samples already cited in the literature. ${ }^{9-11}$ Several chemical methods might be used to disperse catalysts used in the CNT growth on VCarbon substrates. The composite can be submitted to chemical treatments for purification or, yet, incorporation of chemical species able to reduce the work functions of CNTs. 


\section{ACKNOWLEDGMENTS}

J.M.R. and E.Y.M thank the CAPES (Project No. BEX 0278/04-1), CNPq (Rede de Nanotubos de Carbono, Instituto do Milênio), and FAPESP. J.M.R. also thanks the University of Surrey for their hospitality during his sabbatical leave.

${ }^{1}$ R. Saito, G. Dresselhaus, and M. S. Dresselhaus, Physical Properties of Carbon Nanotubes (Imperial College, London, 1998).

${ }^{2}$ S. R. P. Silva, J. D. Carey, G. Y. Chen, D. C. Cox, R. D. Forrest, and C. H. P. Poá, IEE Proc.: Circuits Devices Syst. 151, 489 (2004).

${ }^{3}$ K. A. Dean and B. R. Chalamala, Appl. Phys. Lett. 75, 3017 (1999).

${ }^{4}$ L. Zhong, R. P. G. Wang, W. A. de Heer, and P. Poncharal, Appl. Phys. Lett. 80, 856 (2002).

${ }^{5}$ J. M. Bonard, C. Klinke, K. A. Dean, and B. F. Coll, Phys. Rev. B 67, 115406 (2003).

${ }^{6}$ R. C. Smith, J. D. Carey, D. C. Cox, and S. R. P. Silva, Appl. Phys. Lett. 89, 063111 (2006); R. C. Smith, D. C. Cox, and S. R. P. Silva, ibid. 87,
103112 (2005).

${ }^{7}$ M. Sveningsson, R. E. Morjan, O. Nerushev, and E. E. B. Campbell, Carbon 42, 1165 (2004).

${ }^{8}$ J. M. Rosolen, C. H. P. Poá, S. Tronto, M. S. Marchesin, and S. R. P. Silva, Chem. Phys. Lett. 424, 151 (2006).

${ }^{9}$ J. M. Rosolen, S. Tronto, M. S. Marchesin, E. C. Almeida, N. G. Ferreira, C. H. P. Poá, and S. R. P. Silva, Appl. Phys. Lett. 88, 083116 (2006).

${ }^{10}$ J. M. Rosolen, E. Y. Matsubara, M. S. Marchesin, S. M. Lala, L. A. Montoro, and S. Tronto, J. Power Sources 162, 620 (2006).

${ }^{11}$ L. A. Montoro and J. M. Rosolen, Carbon 44, 3293 (2006).

${ }^{12}$ W. Zhu, C. Bower, O. Zhou, G. Kochanski, and S. Jin, Appl. Phys. Lett. 75, 873 (1999)

${ }^{13}$ C. H. Poa, S. R. P. Silva, P. C. P. Watts, W. K. Hsu, H. W. Kroto, and D. R. M. Walton, Appl. Phys. Lett. 80, 3189 (2002).

${ }^{14}$ P. C. P. Watts, S. M. Lyth, S. J. Henley, and S. R. P. Silva, J. Nanosci. Nanotechnol. 8, 2147 (2008).

${ }^{15}$ J. D. Carey, C. H. Poa, R. D. Forrest, A. P. Burden, and S. R. P. Silva, J. Vac. Sci. Technol. B 18, 1051 (2000). 